\title{
Expanding lacunae causing triventricular hydrocephalus
}

\section{Report of two cases}

\section{Mario Mascalchi, M.D., Ph.D., Fabrizio Salvi, M.D., Ph.D., Umberto Godano, M.D., Marco Nistri, M.D., Rosanna Taiuti, M.D., Michela Tosetti, Ph.D., Natale Villari, M.D., and Fabrio Calbucci, M.D.}

Sezione di Radiodiagnostica, Dipartimento di Fisiopatologia Clinica, Clinica Neurologica, Università di Firenze, Florence, Italy; Divisione di Neurologia, Divisione di Neurochirurgia, Dipartimento di Scienze Neurologiche, Ospedale Bellaria, Bologna, Italy; and IRCCS Stella Maris, Pisa, Italy

Two patients are reported in whom the presence of triventricular hydrocephalus and aqueductal obstruction or stenosis due to multiple expanding lacunae in the mesencephalothalamic region possibly corresponds to abnormally dilated perivascular spaces. Placement of a ventriculoperitoneal cerebrospinal fluid (CSF) shunt in one patient and the performance of a third ventricle cisternotomy in the other reversed the hydrocephalic syndrome, but did not modify the complex neuroophthalmological disturbance and rubral tremor presumably related to the compressive effects of the lacunae on adjacent parenchyma. In one patient the number and size of the lacunae were increased 4 years after CSF shunt placement. A review of the literature revealed two cases in which magnetic resonance imaging demonstrated a similar, poorly understood pathological condition.

Key Words * brain lacunae * hydrocephalus * mesencephalon * magnetic resonance imaging * proton magnetic resonance spectroscopy

Expanding lacunae are uncommon cavities of the brain that are filled with cerebrospinal fluid (CSF). The cause is unclear, although they are possibly a result of abnormally dilated perivascular (Virchow-Robin) spaces that exert a mass effect on adjacent parenchyma and typically bulge into the ventricular cavities.[19] Because they have only been described by pathologists in three cases, $[4,7,18]$ the clinical correlates of expanding lacunae are unclear. Magnetic resonance (MR) imaging enables an in vivo demonstration of expanding lacunae and contributes to their differential diagnosis, which includes other brain cysts.[10,11,17]

In the current report we describe two patients in whom mesencephalothalamic expanding lacunae were observed on MR imaging and in whom the referring complaints were related to triventricular hydrocephalus that was caused by obstruction and stenosis of the cerebral aqueduct and compression of adjacent brain parenchyma. 


\section{CASE REPORTS}

\section{Case 1}

This 58-year-old woman with no significant clinical history was admitted to our hospital in August 1993 because of gait disturbance and tremor of the right arm. The symptoms, which appeared 3 months earlier, were unresponsive to antiparkinsonian drugs and were accompanied by urinary urgency and sudden falls without loss of consciousness.

Examination. The patient's neurological examination showed a wide-based short-stepped gait, bradykinesia, rigidity of the right arm and leg, and increased deep tendon reflexes that were more pronounced on the right side without limb weakness. Nystagmus in the extreme-lateral gaze, limitation of upward eye movements (Parinaud's syndrome), lid retraction, and deficit of convergence featuring a dorsal midbrain syndrome[14] were also present. Finally, a 5- to 6-Hz tremor of the right hand appeared when the patient sustained posture and when the hand was activated. Electromyography of the carpal extensor and flexor muscles showed alternating and synchronous contractions that were consistent with a mixed-pattern tremor caused by damage to the left red nucleus.[1] The patient's score on the Mini-Mental State Examination (MMSE) was 24/30, which is at the lower limit of the normal range. All findings of routine blood laboratory examinations were normal, and testing for Treponema pallidium hemagglutination and syphilis proved negative.

Cranial computerized tomography (CT) scanning revealed triventricular hydrocephalus with periventricular hypodensity and effaced cerebral cortical sulci. No calcification was present. Cranial MR imaging (Fig. 1A and B) confirmed the findings of the CT scanning and demonstrated, in the mesencephalothalamic region, multiple round well-defined areas, ranging in size from 2 to $10 \mathrm{~mm}$, that exhibited a signal intensity identical to CSF and were separated from each other by thin septa of tissue that appeared isointense to the normal brain parenchyma. No enhancement was seen after intravenous administration of contrast agent within or near the lacunae (Fig. 1C). The largest lesions were located in the mesencephalic tegmentum, creating an obstruction of the cerebral aqueduct and displacing the quadrigeminal plate posteriorly. One of these lesions occupied the posterior portion of the left red nucleus. A few tiny lesions of the same type were observed in the medial thalami, and one larger lesion in the right thalamus bulged into the third ventricle. 


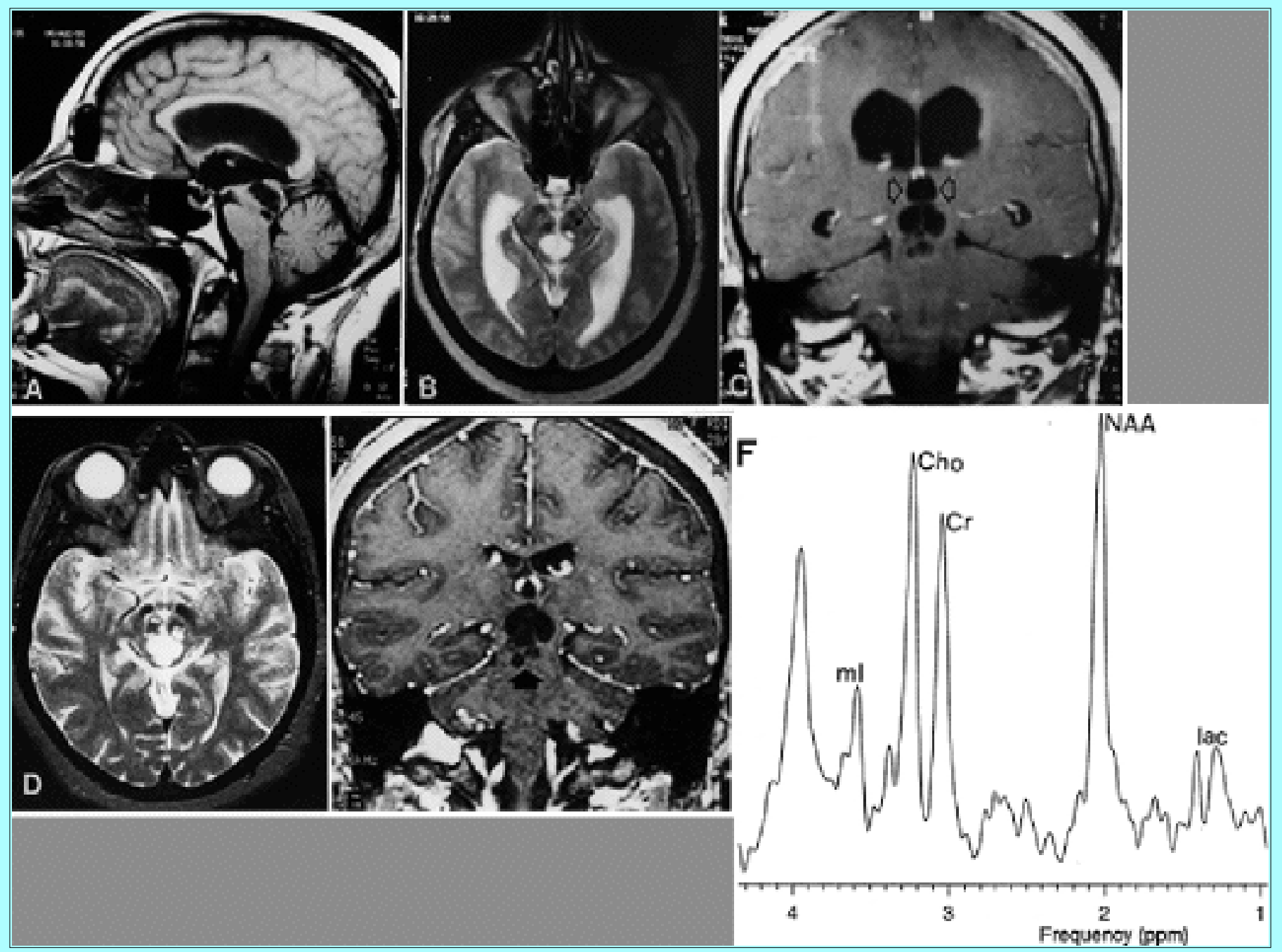

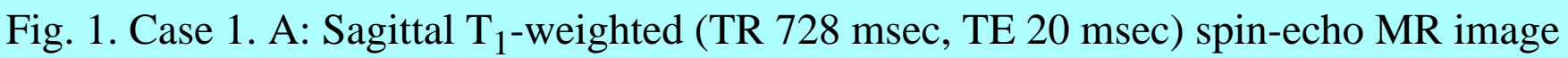
obtained at presentation, revealing multiple lacunae with CSF-like content in the mesencephalic tegmentum, which obstruct the cerebral aqueduct and displace the tectum posteriorly. Note the dilation of the third and lateral ventricles with effacement of the convexity subarachnoid spaces. B: Axial T2-weighted (TR $2280 \mathrm{msec}$, TE $80 \mathrm{msec}$ ) spin-echo MR image demonstrating the CSF-like content of the lacunae in the mesencephalic tegmentum and the lacuna located in the left red nucleus (arrow). C: Coronal $\mathrm{T}_{2}$-weighted (TR $400 \mathrm{msec}$, TE $20 \mathrm{msec}$ ) spin-echo image obtained after intravenous administration of $0.1 \mathrm{mmol} / \mathrm{kg}$ gadolinium-diethylenetriamine pentaacetic acid (Gd-DTPA). No contrast enhancement of the lacunae can be observed. Note the conspicuous dilation of

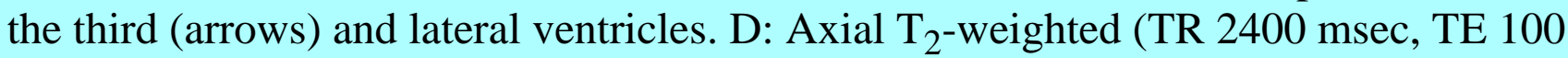
msec) spin-echo MR image obtained 51 months after the ventriculoperitoneal CSF shunt had been placed. Increases in the number of lacunae in the mesencephalic tegmentum and the size of the lacuna in the left red nucleus are evident. $\mathrm{E}$ : Coronal $\mathrm{T}_{1}$-weighted gradient-echo (TR $24 \mathrm{msec}$, TE $9 \mathrm{msec}$, flip angle 45 ) MR image obtained after administration of $0.1 \mathrm{mmol} / \mathrm{kg}$ of Gd-DTPA at the same time as the image shown in D. There is a lack of contrast enhancement of the lacunae, but their increased size (arrowhead) and the disappearance of hydrocephalus is evident. F: Proton MR spectroscopy obtained using the stimulated echo-acquisition method (TR $2000 \mathrm{msec}$, TE $30 \mathrm{msec}$ ) in the central mesencephalon, encompassing the expanding lacunae showing increased lactate (lac) with 
normal peaks of NAA, Cr, Cho, and $\mathrm{mI}$.

Abdominal ultrasonography yielded negative findings, and no focal lesion was seen on chest X-ray films.

Preoperative Treatment and Shunt Placement. Two weeks after admission the patient experienced a severe headache and malaise, which was successfully treated with an intravenous infusion of $18 \%$ mannitol $(280 \mathrm{ml})$. The next day, an external right transfrontal CSF shunt was placed and an intraventricular infusion test was performed. The patient's basal intraventricular CSF pressure was 14 $\mathrm{mm} \mathrm{Hg}$ (normal < $15 \mathrm{~mm} \mathrm{Hg}$ ). Infusion of $10.4 \mathrm{ml}$ saline resulted in a CSF pressure of $34 \mathrm{~mm} \mathrm{Hg}$ at 15 minutes (normal $<20 \mathrm{~mm} \mathrm{Hg}$ ). The CSF analysis showed $0.54 \mathrm{~g} / \mathrm{L}$ proteins (normal $0.1-0.45 \mathrm{~g} / \mathrm{L}$ ), 73 $\mathrm{mg} / \mathrm{dl}$ glucose (normal 50-80 mg/dl), and four leukocytes (normal < 10); no tumor cells or microorganisms were observed during the microscopic examination.

A CSF ventriculoperitoneal shunt with a medium-pressure Holter valve was inserted via a left parietooccipital approach.

Postoperative Course. The patient experienced prompt amelioration of her gait difficulties and bradykinesia. In November 1997 neurological examination showed a dorsal midbrain syndrome and rubral tremor of the right hand. The patient denied having any more gait disturbance, urinary urgency, or sudden falls, and her score on the MMSE was 28/30. Cranial MR imaging (Fig. 1D and E) demonstrated resolution of the triventricular hydrocephalus and an increase in the number and size of the mesencephalothalamic lacunae, which failed to enhance after administration of contrast agent. Proton MR spectroscopy was performed using a 1.5-tesla system with a short echo time-stimulated echo acquisition method sequence (TR $2000 \mathrm{msec}$, TE $30 \mathrm{msec}$ ). A single 15 X 15 X 15-mm voxel was localized in the central midbrain that encompassed the lacunae and demonstrated an increase in lactate and the following ratios of normal metabolites: $\mathrm{N}$-acetyl aspartate (NAA)/creatine $(\mathrm{Cr}), 1.11$; choline (Cho)/Cr 1.12; and myoinositol (mI)/Cr, 0.79 (Fig. 1F). These ratios were similar to those measured in five healthy volunteers who were examined using the same MR system, sequence, voxel size, and location (NAA/Cr $1.19 \pm 0.08$; Cho/Cr $1.06 \pm 0.09$; and mI/Cr $0.84 \pm 0.12)$.

\section{Case 2}

This 55-year-old man presented with a 1-year history of memory loss and gait unsteadiness.

Examination. Neurological examination revealed ataxic gait, a positive Romberg's sign, and nystagmus in the extreme-lateral gaze. Left-eye exotropia in the primary position, "wall-eye" in the upward gaze,[14] and impaired convergence were present. The fundus was normal. Neuropsychological evaluation showed slowness in performing a simple task, a frontal lobe syndrome, and mild memory loss; his MMSE score was 22/30. Cranial CT scanning revealed triventricle hydrocephalus and multiple nonenhancing cysts that appeared isodense to CSF in the mesencephalic tegmentum and the medial portion of the thalami, which were more extensive on the left side. No calcification was seen. Blood tests yielded normal results. Abdominal ultrasonography did not reveal any cystic lesion in the liver, and the patient's chest X-ray film was normal. Cranial MR imaging (Fig. 2A) confirmed the CSF-like content of the cysts, which were surrounded by normal brain parenchyma, and determined a marked stenosis of the upper outlet of the cerebral aqueduct. No contrast enhancement was seen (Fig. 2B). Single (20 X 20 X 20 $\mathrm{mm}$ )-voxel proton MR spectroscopy was performed using a point-resolved spectroscopy sequence (TR $2000 \mathrm{msec}$, TEs 135 and $270 \mathrm{msec}$ ) in the left mesencephalothalamic junction. This revealed an increase in the lactate peak, exhibiting the characteristic phase reversal with a $135-\mathrm{msec} \mathrm{TE}$, without significant 
abnormalities in the NAA/Cr (2.02) and Cho/Cr (1.50) ratios (Fig. 1C) as compared with those measured in the thalamus of three healthy volunteers (NAA/Cr $2.39 \pm 0.42$; Cho/Cr $1.30 \pm 0.26$ ).

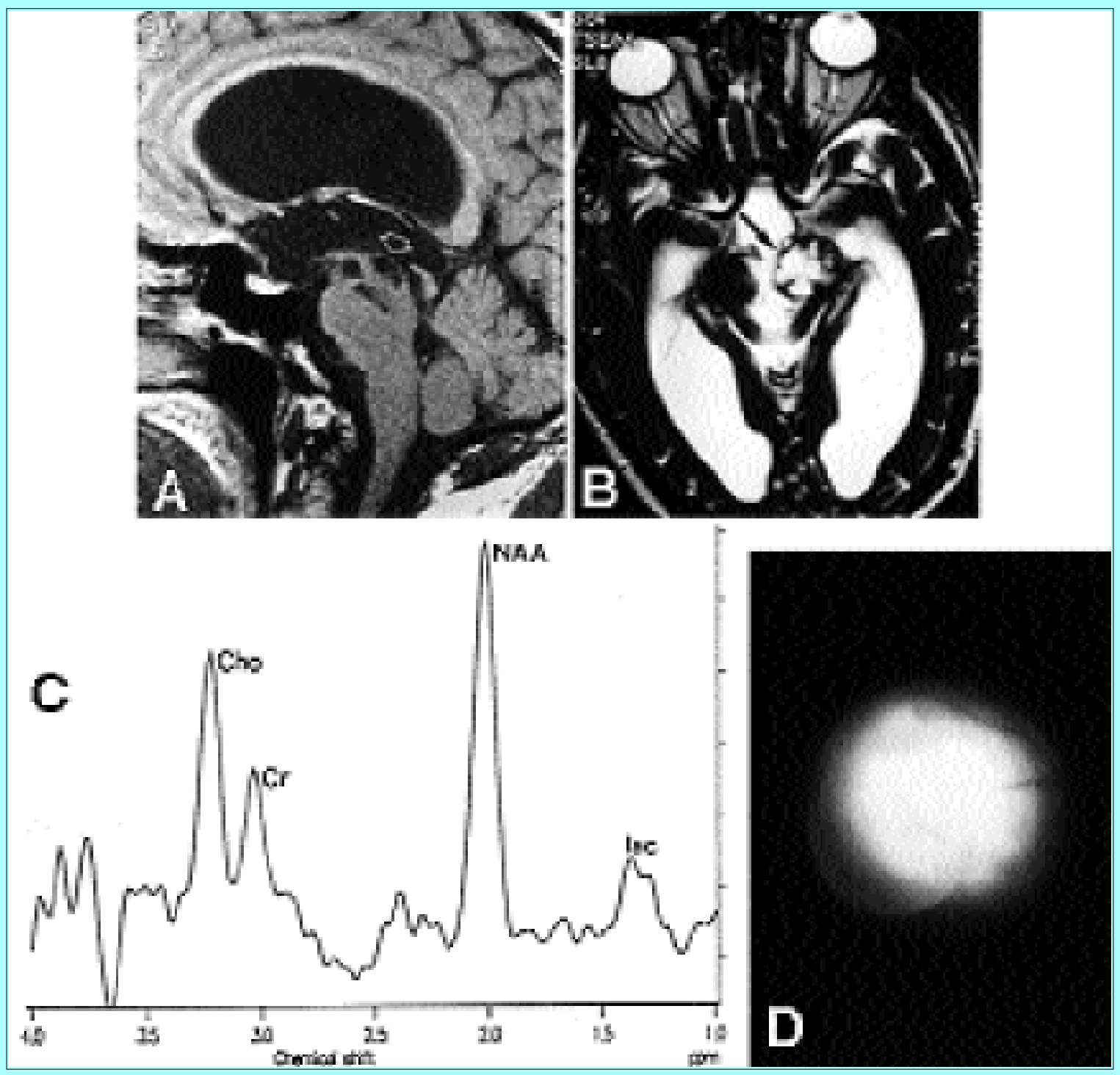

Fig. 2. Case 2. A: Sagittal T1-weighted (TR $500 \mathrm{msec}$, TE $20 \mathrm{msec}$ ) spin-echo MR image obtained at presentation, revealing dilation of the lateral and third ventricles and stenosis of the upper outlet of the cerebral aqueduct due to small CSF-filled cysts in the mesencephalic

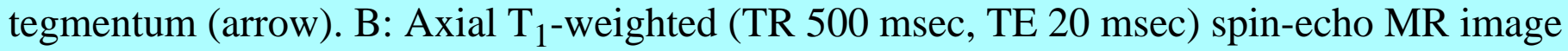
obtained after intravenous administration of Gd-DTPA, demonstrating nonenhancing CSF cysts in the left cerebral peduncle and the central mesencephalon. C: Proton MR spectroscopy obtained using the point-resolved spectroscopy technique (TR $2000 \mathrm{msec}$, TE $270 \mathrm{msec}$ ) in the left mesencephalothalamic junction encompassing the expanding lacunae, revealing an increase in lactate (lac) with normal peaks of NAA, Cr, and Cho. D: Endoscopic view of the third ventricle demonstrating balloonlike translucent left ventricular wall protrusion, corresponding to an expanding lacuna bulging within the ventricle.

Endoscopic Treatment and Findings. The patient underwent endoscopic third ventricle cisternotomy via a right transfrontal approach. During the procedure, balloonlike translucent bulgings of the third ventricle walls were observed (Fig. 2D). The CSF was colorless and clear and contained $0.2 \mathrm{~g} / \mathrm{L}$ proteins, $43 \mathrm{mg} / \mathrm{dl}$ glucose, and no leukocytes. No microorganisms or tumor cells were observed during the CSF 
cytological examination, and the results of enzyme-linked immunosorbent assay for echinococcosis were negative.

Postoperative Course. A neurological examination performed 6 months after surgery showed normal gait and a negative Romberg's sign. The patient was more attentive, collaborative, and rapid during the neuropsychological examination, and his score on the MMSE was 29/30. Cranial MR imaging was unchanged except for the third ventricle cisternotomy.

\section{DISCUSSION}

Based on pathological features, Poirier and Derouesné[19] classified brain lacunae into three fundamental types: Type I lacunae correspond to lacunar infarction; Type II lacunae correspond to small intraparenchymal hematomas; and Type III (expanding) lacunae correspond to dilated perivascular (Virchow-Robin) spaces. The latter are lined by a single stratum of epithelium-like flat cells and contain a small artery or arteriole. The brain parenchyma adjacent to the expanding lacunae exhibits only limited gliosis, spongiosis, edema, or demyelination. Magnetic resonance imaging demonstrates enlarged perivascular spaces as tiny-to-large CSF-filled cavities with well-defined smooth margins that are surrounded by normal brain parenchyma and display no enhancement after administration of contrast agent.[11,17] The typical distribution of the perivascular spaces visible on MR imaging includes the basal ganglia, the cerebral white matter, and the midbrain, conforming to the path of the penetrating arteries. In particular, in $20 \%$ of patients in whom MR imaging was performed, tiny perivascular spaces were identified at the junction of the substantia nigra and cerebral peduncle, where the penetrating branches of the collicular or accessory collicular arteries are located.[8] These dilated mesencephalic perivascular spaces had no clinical correlate.

The large CSF-filled cavities in our patients had a distribution similar to the asymptomatic dilated perivascular spaces described earlier and to those reported in a case of expanding lacunae verified by pathological investigation.[18] This is consistent with the view that mesencephalothalamic expanding lacunae are normal perivascular spaces undergoing abnormally conspicuous dilation.

In our patients, diagnosis of mesencephalothalamic expanding lacunae was based mainly on CT and MR imaging findings. Although MR imaging is suitable to differentiate the three fundamental types of brain lacunae, $[5,8,11]$ a differential diagnosis that includes other intracranial cystic lesions in the same location, in particular those due to parasites (echinococcosis, cryptooccocosis, and cysticercosis), has to be considered.[10] Cryptococcus neoformans has a predilection for the brain perivascular spaces,[2,22] but cryptococcosis usually occurs against the background of acquired immunodeficiency syndrome and is associated with CSF abnormalities and extensive changes and contrast enhancement of adjacent brain parenchyma on imaging studies, which were absent in our patients. Lack of calcification and contrast enhancement is also unusual for cysticercosis, which has a predilection for the ventricular or subarachnoid spaces.[13] Although calcifications are rare and contrast enhancement is inconstant and mild in brain hydatidosis, Echinococcus granulosus usually produces a solitary large unilocular cyst with fluid that appears hyperintense to CSF on proton density-weighted MR images.[12,15] Moreover, immunoreactions for hydatidosis were negative in the CSF of one of our patients.

Additional nonneoplastic intracranial cysts to be differentiated from mesencephalothalamic expanding lacunae are ependymal, neuroepithelial, and arachnoid cysts. However, these cysts are not multiple as a rule, their content may not appear the same as CSF on MR imaging, and their location may not conform to the path of the penetrating arteries. $[8,10,11,17]$ 
Proton MR spectroscopy is increasingly used as a supplement to MR imaging to characterize cystic lesions of the brain due to tumor, abscess, and parasitic infestations. $[6,12]$ In parasitic cysts a wide range of abnormal metabolites, including alanine, acetate, succinate, and pyruvate, have been identified within cystic fluid in addition to a variable amount of lactate and the complete absence of normal brain metabolites.[6,12] This pattern is similar to that observed in brain abscesses,[6] but different from that found in cystic tumors in which variable amounts of lactate and lipids are present together with a reduction in NAA and an increase in Cho due to inclusion of abnormal pericystic parenchyma within the region of interest.[6] In both of our patients, we included expanding lacunae and surrounding normal-appearing brain parenchyma in the region of interest for proton spectroscopy. The increased levels of lactate without changes in the ratios of $\mathrm{NAA} / \mathrm{Cr}, \mathrm{Cho} / \mathrm{Cr}$, and $\mathrm{mI} / \mathrm{Cr}$ are consistent with the increased content of normal CSF within the expanding lacunae[3,16,23] and reflect the paucity of tissue alterations, if any, in the surrounding parenchyma.

Clinical correlates of expanding lacunae are uncertain. In the 54-year-old women described by Poirier, et al.,[18] expanding lacunae were located in the mesencephalic tegmentum and thalami. Although CT scanning, ventriculography, and pathological studies demonstrated triventricular hydrocephalus and obstruction of the cerebral aqueduct by the expanding lacunae, the patient's mental deterioration was thought to be due to the local effect of expanding lacunae in the thalamus (thalamic dementia). However, in few years a progressive gait disturbance and total sphincteric incontinence developed in the patient, indicative of the triad of normal-pressure hydrocephalus syndrome. Moreover, in the same patient, a ventriculocisternotomy resulted in a transient improvement in symptoms a few days before his death.

A review of the literature yielded only two other cases of expanding lacunae confirmed by pathological examination,[4,7] in which no clinical correlates of the lesions, located near the caudate and dentate nuclei, respectively, can be found in the brief case history provided. As well, no clinical correlate was found by Ogawa, et al.,[17] in two cases of dilated perivascular spaces demonstrated by MR imaging in cerebral hemispheric white matter, although the results of a biopsy sample confirmed one of these cases. Also, in a 42-year-old man described by Homeyer, et al.,[10] in whom a diagnosis of expanding lacunae based on MR imaging and negative laboratory examinations for parasites had been made, the expanding lacunae had a mesencephalothalamic distribution, causing stenosis of the aqueduct and triventricle hydrocephalus. The clinical features in that case included headache, blurred vision, diplopia, paresthesias to the upper limbs, skew deviation, and Parinaud's syndrome. The patient's symptoms transiently regressed after CSF shunt placement and evacuatory CSF lumbar puncture; however, neither of these treatments modified the MR imaging findings. The clinical presentation in our patients included gait disturbance of the parkinsonian and ataxic types, mental deterioration, urinary urgency, and falling spells, which were clearly explained by the triventricular obstructive hydrocephalus demonstrated on MR imaging. $[9,14,20]$ These were successfully treated by CSF ventriculoperitoneal shunt placement and third ventricle cisternotomy.

Based on our cases and two others in the literature,[10,18] expanding lacunae in the mesencephalic tegmentum can be regarded as an additional rare cause of benign aqueductal obstruction and noncommunicating normal-pressure hydrocephalus, in addition to the aqueductal stenosis emphasized by Vanneste and Hyman.[21]

Although one can suppose that expanding lacunae do not cause symptoms per se, the persistence of dorsal mesencephalic syndrome and rubral tremor after CSF shunt placement in our first patient and the complex neuroophthalmological picture observed after ventriculostomy in our second patient indicate 
that expanding lacunae may cause focal neurological dysfunction.

The etiopathogenesis of expanding lacunae is obscure, and three main hypotheses have been proposed. The coincidence of their distribution according to territories of the paramedian mesencephalothalamic perforating arteries was noted by Poirier, et al.,[18] who observed segmental necrotizing vasculitis in the proximal portion of one of these vessels in their case. These authors proposed that expanding lacunae are dilated perivascular spaces caused by a disorder of the permeability of the arterial wall that is possibly secondary to vasculitis. The lack of contrast enhancement of the lesions and nearby parenchyma on MR imaging in our cases and in the case reported by Homeyer, et al.,[10] however, is at variance with this hypothesis. Moreover, vasculitis was not present in the two other cases of expanding lacunae that have been verified by pathological investigation,[4,7] in which multiple cerebral infarctions and sclerohyalinotic vascular changes secondary to long-standing arterial hypertension were documented. In the case reported by Homeyer, et al.,[10] and in our cases, no brain vascular lesion, vascular risk factor, or evidence of systemic vasculitis was present.

Based on the association of mesencephalothalamic expanding lacunae and hydrocephalus, $[7,18]$ Derouesné and colleagues[7] suggested that the expanding lacunae are dilated perivascular spaces that result from difficult drainage of brain interstitial fluid into the ventricles due to increased pressure of intraventricular CSF. The increased number and size of the expanding lacunae in our first patient, after resolution of hydrocephalus with a CSF shunt, confutes this hypothesis. Finally, Homeyer, et al.,[10] hypothesized that the abnormal dilation of the mesencephalothalamic perivascular spaces may be related to a disturbance in brain interstitial fluid drainage, possibly secondary to obstruction of lymphatic drainage of the brain occurring along routes adjacent to cranial and spinal nerves. This hypothesis might be in agreement with the follow-up MR imaging findings in our first patient; however, it awaits further confirmation.

\section{CONCLUSIONS}

Our observations indicate that the CT and MR imaging and proton MR spectroscopy features found in our cases (sites corresponding to the path of the penetrating arteries, multiplicity, no calcification, fluid signal identical to CSF on all MR imaging sequences, no signal changes in adjacent brain parenchyma, no contrast enhancement, no abnormal metabolites although lactate is present with normal NAA/Cr and $\mathrm{Cho} / \mathrm{Cr}$ ratios) in conjunction with negative laboratory findings for parasites may allow the diagnosis of expanding lacunae. When located in the mesencephalothalamic region, expanding lacunae can become symptomatic, producing either triventricular hydrocephalus due to aqueductal obstruction or focal symptoms and signs due to the compressive effect on adjacent brain parenchyma. Placement of a CSF shunt or ventriculocisternotomy can treat the hydrocephalus syndrome but does not modify the focal symptoms and signs or arrest lesion growth.

\section{References}

1. Adams RA, Victor M (eds): Principles of Neurology, ed 3. New York: McGraw-Hill, 1985

2. Andreula CF, Burdi N, Carella A: CNS cryptoccocosis in AIDS: spectrum of MR findings. J Comput Assist Tomogr 17:438-441, 1993

3. Bell JD, Brown JCC, Sadler PJ, et al: High resolution proton nuclear magnetic resonance studies of human cerebrospinal fluid. Clin Sci 72:563-570, 1987 
4. Benhaïem-Sigaux N, Gray F, Gherardi R, et al: Expanding cerebellar lacunes due to dilatation of the perivascular space associated to Binswanger's subcortical arteriosclerotic encephalopathy. Stroke 18:1087-1092, 1987

5. Challa VR, Moody DM: The value of magnetic resonance imaging in the detection of type II hemorrhagic lacunes. Stroke 20:822-825, 1989

6. Chang KH, Song IC, Kim SH, et al: In vivo single-voxel proton MR spectroscopy in intracranial cystic masses. AJNR 19:401-405, 1998

7. Derouesné C, Gray F, Escourolle R, et al: Expanding cerebral lacunae in a hypertensive patient with normal pressure hydrocephalus. Neuropathol Appl Neurobiol 13:309-320, 1987

8. Elster AD, Richardson DN: Focal high signal on MR scans of the midbrain caused by enlarged perivascular spaces: MR-pathologic correlation. AJR 156:157-160, 1990

9. Haan J, Jansen ENH, Oostrom J, et al: Falling spells in normal pressure hydrocephalus: a favourable prognostic sign? Eur Neurol 27:216-220, 1987

10. Homeyer P, Cornu P, Lacomblez L, et al: A special form of cerebral lacunae: expanding lacunae. J Neurol Neurosurg Psychiatry 61:200-202, 1996

11. Jungreis CA, Kanal E, Hirsch WL, et al: Normal perivascular spaces mimicking lacunar infarction: MR imaging. Radiology 169:101-104, 1988

12. Kohli A, Gupta RK, Poptani H, et al: In vivo proton magnetic resonance spectroscopy in a case of intracranial hydatis cyst. Neurology 45:562-564, 1995

13. Kramer LD, Locke GE, Byrd SE, et al: Cerebral cysticercosis: documentation of natural history with CT. Radiology 171:459-462, 1989

14. Leigh RJ, Zee DS: The Neurology of Eye Movements, ed 2. Philadelphia: FA Davis, 1991

15. Mascalchi M, Ragazzoni A, Dal Pozzo G: Pontine hydatis cyst in association with an acoustic neurinoma: MR appearance in an unusual case. AJNR 12:78-79, 1991

16. Matthews PM, Pioro E, Narayanan S, et al: Assessment of lesion pathology in multiple sclerosis using quantitative MRI morphometry and magnetic resonance spectroscopy. Brain 119:715-722, 1996

17. Ogawa T, Okudera T, Fukasawa H, et al: Unusual widening of Virchow-Robin spaces: MR appearance. AJNR 16:1238-1242, 1995

18. Poirier J, Barbizet J, Gaston A, et al: Démence thalamique. Lacunes expansives du territoire thalamo-mésencéphalique paramédian. Hydrocéphalie par sténose de l'aqueduct de Sylvius. Rev Neurol 139:349-358, 1983

19. Poirier J, Derouesné C: Le concept de lacune cérébrale de 1838 a nos jours. Rev Neurol 141:3-17, 1985

20. Sypert GW, Leffman H, Ojemann GA: Occult normal pressure hydrocephalus manifested by parkinsonian-dementia complex. Neurology 23:234-238, 1973 
21. Vanneste J, Hyman R: Non-tumoural aqueduct stenosis and normal pressure hydrocephalus in the elderly. J Neurol Neurosurg Psychiatry 49:528-535, 1986

22. Wehn SM, Heinz ER, Burger PC, et al: Dilated Virchow-RObin spaces in cryptococcal meningitis associated with AIDS: CT and MR findings. J Comput Assist Tomogr 13:756-762, 1989

23. Wevers RA, Engelke U, Wendel U, et al: Standardized method for high-resolution 1H-NMR of cerebrospinal fluid. Clin Chem 41:744-751, 1995

Manuscript received December 28, 1998.

Accepted in final form April 30, 1999.

Address reprint requests to: Mario Mascalchi, M.D., Ph.D., Sezione di Radiodiagnostica, Dipartimento di Fisiopatologia Clinica, Università di Firenze, Viale Morgagni 85, 50134 Florence, Italy. email: m.mascalchi@dfc.unifi.it. 